\title{
Downlink Macrodiversity in Cellular Network - A Fluid Analysis
}

\author{
Jean-Marc Kelif ${ }^{1}$ and Eitan Altman ${ }^{2}$ \\ ${ }^{1}$ France Telecom Research and Development Issy Moulineaux, France \\ ${ }^{2}$ INRIA Sophia Antipolis, France \\ jeanmarc.kelif@orange-ftgroup.com, eitan.altman@sophia.inria.fr
}

\begin{abstract}
This paper proposes an analytical study of the downlink macrodiversity. Considering two macrodiversity links, we first show that the downlink macrodiversity induces a specific load in the cell. We establish an explicit expression of this one, and show that macrodiversity increases the total cell's load. The network's fluid analysis we propose enables to calculate the load of a cell and to quantify the impact of macrodiversity. We show that macrodiversity decreases the capacity of a cell. We generalize the analysis, considering a macrodiversity with a great number of base stations of the network.
\end{abstract}

\section{Introduction}

The studies related to macrodiversity were mainly done for the uplink [7] [8]. Hanly [7] described macrodiversity as a scheme in which the cellular structure of a wireless communication network is removed and user is jointly decoded by all receivers in the network. Hiltunen and de Bernardi [4] developed a downlink analysis of the macrodiversity to estimate a CDMA network's capacity. Their analysis considers the macrodiversity use to maintain the SIR target of mobiles in soft/softer handover with two base stations. It is well known that downlink macrodiversity induces an extra load that can be considered as the "price" for obtaining the macrodiversity gain. In this paper we demonstrate why there is an extra load. We give its analytical expression and quantify with a high accuracy the impact of macrodiversity. Afterward, we propose a model which allows calculating analytically that extra load. This model considers the network as a continuum of base stations and allows calculating the influence of any mobile in a cell, whatever its position. For clarity of presentation, this paper is focused on CDMA networks. However, the analysis we develop can be used for other technologies such as OFDMA (see remark at the end of the section 2.1).

The paper is organized as follows. In Section 2 we introduce the interference factor $f_{i}^{D L}$ which characterizes the "weight" of the network, on a given cell. We 
show that mobiles in macrodiversity induce a positive specific load $L_{M D}$. In Section 3, we express the cell's load, using a fluid model of the network [9][11]. This approach considers the network as a continuum. It can be applied to any frequency reuse 1 networks, such as OFDMA or CDMA ones. In Section 4, we establish the macrodiversity decreases a cell's capacity. In Section 5, we generalize the macrodiversity analysis to a whole network, and show it always induces a decrease of the capacity. In Section 6 we conclude.

\section{Cellular network Analysis}

\subsection{Network analysis}

We use the model similar to [4]. Let us consider a mobile connected to the base station $b$ of a network of $N_{B S}$ base stations, each $B S_{j}$ defining a cell $j$. We express that the Signal to Interference Ratio (SIR) received by a mobile has to be at least equal to a minimum threshold target value $\gamma_{i}$ [4] [5]. Each mobile uses only one service. Using the equation of the transmitting traffic channel power [4] for the downlink, the following condition has to be satisfied:

$$
\frac{P_{i b} g_{i b}}{\alpha I_{\text {int }}+I_{\text {ext }}+\text { Noise }} \geq \gamma_{i}
$$

where $P_{i b}$ is the useful transmitting power coming from the base station $b$ towards the mobile $\mathrm{i}$ belonging to the base station $b$, and $g_{i b}$ is the pathloss between the base station $\mathrm{b}$ and the mobile $i, I_{\mathrm{int}}$ is the interferences due to the common channels and the traffic channels of the other mobiles located in the cell $b$, and $I_{\text {ext }}$ is the interferences due to the other base stations of the network, $\gamma_{i}$ represents the level of the signal to interference ratio target for the service used by the mobile $i$ for the downlink, Noise stands for the level of noise floor at the mobile receiver, and $\alpha$ the orthogonality factor, and $g_{j, i}$ the pathloss between the mobile $i$ and the base station $j$. Introducing $P_{b}$ the total transmitting power of the base station $b$, including the common channels assumed as orthogonal, and $\beta_{i}=\frac{\gamma_{i}}{1+\alpha \gamma_{i}}$. For each mobile $i$ belonging to the cell $\mathrm{b}$, we define the parameter $f_{i}^{D L}$, as the ratio between the total power $P_{\text {ext }}$ received by the mobile $i$ coming from the other base stations of the network to the total power $P_{\text {int }}$ received by its serving base station b:

$$
f_{i}^{D L}=\frac{P_{e s t}}{P_{\text {int }}}=\frac{1}{P_{b} g_{i b}} \sum_{j=1, j \neq b}^{N_{B S}} P_{j} g_{i j}
$$


We express from (2.1) the minimum needed traffic channel transmitting power as:

$$
P_{i b} g_{i b}=\beta_{i}\left(\alpha P_{b} g_{i b}+f_{i}^{D L} P_{b} g_{i b}+\text { Noise }\right)
$$

\section{Remark}

Though our analysis is focused on CDMA networks, the model we develop is still valid for cellular technologies without internal interference, providing that $I_{\text {int }}=0$. It can be applied, in particular, to frequency reuse 1 networks based on other technologies, such as OFDMA.

\subsection{Downlink macrodiversity analysis}

\section{Base station transmitting power}

Our approach is inspired by [4]. The downlink macrodiversity allows a mobile to use the signals received from more than one base station to reach the requested SIR target. We establish the analytical expression of the cell's load, taking into account the macrodiversity with two base stations, and show that the macrodiversity always increases the load. A mobile $i$ in macrodiversity is connected to two base stations $b$ and $l . b$ is defined to be the base station with larger SIR. We express that the power control tries to maintain the SIR target $\gamma_{i}$. We denote $\gamma_{i, l}$ and $\gamma_{i, b}$ the SIR received by mobile $i$ coming from base stations $l$ and $b$, and we assume that:

$$
\Omega_{i}=\frac{\gamma_{i, l}}{\gamma_{i, b}} \leq 1
$$

is a constant. The SIR target is:

$$
\gamma_{i}=\gamma_{i, b}+\gamma_{i, l}
$$

Considering a mobile belonging to the $\mathrm{BS} b$ and in macrodiversity with the BS $l$, and introducing $\kappa_{i}=\frac{\gamma_{i}}{1+\Omega_{i}\left(1+\gamma_{i}\right)+\alpha \gamma_{i}}$ (2.4c), the expression (2.3) becomes, for a mobile $i$ in macrodiversity:

$$
P_{i b}=\kappa_{i}\left(\alpha P_{b}+f_{i}^{D L} P_{b}+\text { Noise } / g_{i b}\right)
$$

Let there be $N$ mobiles in a cell $b, M$ among them are in macrodiversity with the BS $l$. As a consequence, in the macrodiversity zone we can consider $M$ mobiles belonging to the $\mathrm{BS} b$ and $P$ mobiles belonging to the $\mathrm{BS} l$ in macrodiversity with $b$. These last ones also receive a signal from the BS $b$. Denoting $P_{C C H}$ the power dedicated to the common channels, the total transmitting power of the base station $b$ can be expressed as the sum of all the transmitting powers channels: 


$$
P_{b}=\sum_{i=1}^{N-M} P_{i b}+\sum_{j=1}^{M} P_{j b}+\sum_{k=1}^{P} P_{k b}+P_{C C H}
$$

\section{Hypothesis}

For a homogeneous repartition of mobiles in the macrodiversity zone, we can assume that $M=P$. And statistically, due to the homogeneity of the mobiles repartition, the total power dedicated to the mobiles $M$ should be the same as the total power dedicated to the mobiles $P$. So we can write:

$$
\sum_{j=1}^{M} P_{j b}=\sum_{k=1}^{P} P_{k b}
$$

The expression (2.5) can be rewritten:

$$
P_{b}=\sum_{i=1}^{N-M} P_{i b}+2 \sum_{j=1}^{M} P_{j b}+P_{C C H}
$$

The power $P_{C C H}$ dedicated to common channels is assumed as proportional to the power of the base station so we have $P_{C C H}=\varphi P_{b}$. Denoting:

$$
A=\sum_{i}^{N-M} \beta_{i} \text { Noise } / g_{i b}+2 \sum_{j}^{M} \kappa_{j} \text { Noise } / g_{j b}
$$

and

$$
L=\sum_{i=1}^{N-M} \beta_{i}\left(\alpha+f_{i}^{D L}\right)+2 \sum_{j=1}^{M} \kappa_{j}\left(\alpha+f_{j}^{D L}\right)
$$

the total transmitting power of BS $b$ can be deduced from (2.4) and (2.7) and written as:

$$
P_{b}=\frac{A}{1-\varphi-L}
$$

When the number of mobiles in a cell increases, the parameter $L$ increases too. Consequently the transmitting power of BS $b$ increases. $L$ represents the total load of the cell.

\section{Remarks:}

The authors of [4] assumed all links within the active set have the same transmit power. This condition is stronger than our statistical hypothesis $(M=P)$. Furthermore, in [4] the authors consider another strong assumption: all the interference factors have the same value. We consider the exact values of this parameter: this one varies with the position of the mobile in the cell. 


\subsection{Macrodiversity load}

Each mobile in the cell induces a specific load. To analyze the macrodiversity effect, we need to express that one considering mobiles either in macrodiversity or not. The cell's load $L$ (2.8a) can be expressed as:

$$
L=L_{D L}+L_{M D}
$$

The first term:

$$
L_{D L}=\sum_{i=1}^{N} \beta_{i}\left(\alpha+f_{i}^{D L}\right)
$$

takes into account all the mobiles of the cell. It represents the cell's load, for the downlink, in a case where there is no macrodiversity. When a mobile is not in macrodiversity, we notice, from (2.4a), that $\Omega_{i}=0$ and $\kappa_{i}=\beta_{i}$. Introducing:

$$
\sigma_{i}=\frac{\Omega_{i}}{1+\alpha \gamma_{i}}
$$

we can write:

$$
\kappa_{i}=\beta_{i}\left(1-\frac{\sigma_{i}}{1+\sigma_{i}}\right)
$$

We can express:

$$
L_{M D}=\sum_{j=1}^{M} \beta_{j}\left(\frac{1-\sigma_{j}}{1+\sigma_{j}}\right)\left(\alpha+f_{j}^{D L}\right)
$$

This term represents an extra load due to the fact that $M$ mobiles among $N$ are in macrodiversity. Since $0 \leq \sigma_{j}=\frac{\Omega_{j}}{1+\alpha \gamma_{j}} \leq 1$ (2.12), the load $L_{M D}$ is positive.

Expressing the total transmitting power of a BS, our analysis explicitly shows the macrodiversity induces a specific positive load $L_{M D}$ in the cell, increasing its total load. The consequence of this increase is to decrease the capacity (number of mobiles, throughput) of the cell. Considering the analytical expression of $L_{M D}$, our analysis moreover allows to identify the parameters which have an explicit influence on that macrodiversity extra load: The QoS characterized by $\beta_{i}$, the base stations transmitting powers (interference factor $f_{i}^{D L}$ ) and the term $\frac{1-\sigma_{j}}{1+\sigma_{j}}$ characterizes the powers received from the base stations with which a mobile is in macrodiversity. It appears interesting to calculate analytically this extra load. In this aim, we need to express analytically the parameters contributing to $L_{M D}$, and particularly the downlink interference factor $f_{i}^{D L}$. Hereafter we propose an analytical approach which allows to calculate $L_{M D}$. 


\section{Analytical fluid model}

The key modelling step of the model we propose consists in replacing a given fixed finite number of transmitters (base stations or mobiles) by an equivalent continuum of transmitters which are distributed according to some distribution function. We denote it a fluid model [9] [11]. We consider a traffic characterised by a mobile density $\rho_{M S}$ and a network by a base station density $\rho_{B S}$. For a homogeneous network, the downlink interference factor only depends on the distance $r$ between the BS and the mobile. We denote it $f_{r}$. From [9] [11], we have:

$$
f_{r}=\frac{\rho_{B S} \cdot 2 \pi}{(\eta+2) r^{\eta}} \cdot\left[(R-r)^{\eta+2}-\left(2 R_{c}-r\right)^{\eta+2}\right]
$$

\subsection{Load model with macrodiversity}

Using the analytical expression of the interference factor (3.1), and the expressions (2.9) (2.10) and (2.11), we aim to express hereafter the load $L$ of the cell. The parameters $\gamma_{i} \beta_{i}$ and $\kappa_{i}$, which characterize the QoS of the mobiles, depend on the service used by the mobile $i$. We assume that these parameters may also depend on the distance $r$ of the mobile from its serving $\mathrm{BS} b$ and write them $\gamma_{r} \beta_{r} \kappa_{r}$. The parameters $\Omega_{i}$ and $\sigma_{i}$ can be written $\Omega_{r}$ and $\sigma_{r}$. Considering that mobiles use one service we can drop the index $i$. In fact, the providers have the choice to modify the mobiles' QoS for them to be admitted in the cell. And this modification may depend on the position of the mobile. The expression of the total transmitting power of $\mathrm{BS} b$, considering the mobiles located at distances $r$ from the base station $b$ is given by (2.8) .From (2.8a) and (2.8b), we have :

and

$$
A=\sum_{r<R_{i, h}} N_{r} \beta_{r} \frac{\text { Noise }}{g_{b, r}}+2 \sum_{r \geq R_{\text {ith }}} N_{r} \kappa_{r} \frac{\text { Noise }}{g_{b, r}}
$$

$$
L=\sum_{r<R_{\text {th }}} N_{r} \beta_{r}\left(\alpha+f_{r}^{D L}\right)+2 \sum_{r \geq R_{\text {th }}} N_{r} \kappa_{r}\left(\alpha+f_{r}^{D L}\right)
$$

where $N_{r}$ represents the number of mobiles located at a distance $r$ from the BS $b$, $R_{t h}$ is a threshold distance defining the macrodiversity zone: If $r<R_{t h}$ a mobile is not in macrodiversity, and he is in macrodiversity otherwise. We notice moreover that mobiles at a given distance $r$ have the same values of interference factor $f_{r}$ in our analytical model. Considering the network (base stations and mobiles) as a continuum of transmitters characterized by a base station density $\rho_{B S}$ and a mobile density $\rho_{M S}$, we can replace the discrete summations

(3.2) and (3.3) by continuous ones and express the $\operatorname{load} L$ with integrals. Considering a macrodiversity zone with one neighbor (figure 1 , base stations $b$ and $l$ ), the expression of the cell's load (3.3) can be rewritten as: 


$$
L=\int_{-\frac{\pi}{6}}^{+\frac{\pi}{6}} \int_{0}^{R_{t h}} \rho \beta_{r}\left(\alpha+f_{r}\right) \cdot r \cdot d r d \theta+2 \int_{-\frac{\pi}{6}}^{+\frac{\pi}{6}} \int_{R_{t h}}^{R_{C}} \rho \kappa_{r}\left(\alpha+f_{r}\right) \cdot r \cdot d r d \theta
$$

In a network, it is currently assumed that a cell $b$ is surrounded by 6 cells (figure 1). Considering there is a macrodiversity zone with each one (figure 1) and assuming all the macrodiversity zones are identical (figure 1) (homogeneous network), we can write the loads expressions (2.10) and (2.11) as:

$$
L_{D L}=2 \cdot \pi \int_{0}^{R_{C}} \rho \beta_{r}\left(\alpha+f_{r}\right) r \cdot d r
$$

and

$$
L_{M D}=2 . \pi \int_{R_{t h}}^{R_{c}}\left(\frac{1-\sigma_{r}}{1+\sigma_{r}}\right) \rho \beta_{r}\left(\alpha+f_{r}\right) r d r
$$

We showed (Section 2) that the analytical expression of $L_{M D}$ enables to determine the parameters which have an influence on the extra load due to the macrodiversity: the QoS, the BS transmitting powers and the term $\left(\frac{1-\sigma_{r}}{1+\sigma_{r}}\right)$. Another parameter explicitly appears, highlighted by the fluid model: the macrodiversity size' zone characterized by $R_{t h}$.

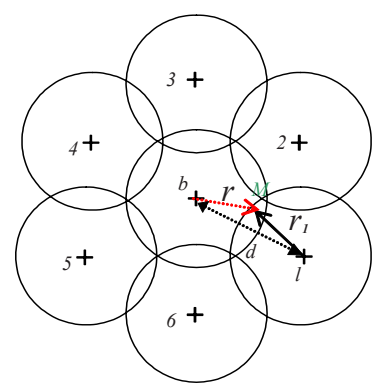

Fig. 1. Cell $b$ sharing macrodiversity zones with its 6 neighbours

\section{Admission control analysis}

\subsection{General Analysis}

For the downlink, we express that the power of the base station $P_{b}$ is limited to a maximum value $P_{\max }$ : the call admission control is based on the probability $P^{D L}$ to satisfy the following relation: $P^{D L}=\operatorname{Pr}\left[P_{b}>P_{\max }\right]$ which can be expressed, using (2.8): 


$$
P^{D L}=\operatorname{Pr}\left(L>1-\varphi-\frac{A}{P_{\max }}\right)
$$

The cell load $L$ is expressed by (2.9) (3.5) and (3.6). To analyze the admission control, we first need to calculate analytically the expressions (3.5) and (3.6) of $L_{D L}$ and $L_{M D}$. In a general case, the QoS dependency with the position of the mobile $\left(\beta_{r}\right)$ in the cell depends on the strategies of the provider: different strategies can be adopted, and for each one that dependency may be different. We will adopt a strategy where the QoS offered to the mobiles do not vary with the position of the mobiles $\left(\beta_{r}=\beta\right.$, and $\left.\sigma_{r}=\sigma\right)$ and write from (3.5) and (3.6):

$$
L_{D L}=2 \pi \rho \oint \int_{0}^{R_{c}}\left(\alpha+f_{r}\right) r d r
$$

and

$$
L_{M D}=2 \pi \rho \beta \int_{R_{t h}}^{R_{c}}\left(\frac{1-\sigma}{1+\sigma}\right)\left(\alpha+f_{r}\right) r d r
$$

We introduce the downlink average interference factor $F_{D L}$ for the whole cell as:

$$
F_{D L}=\frac{1}{S} \int_{0}^{R_{c}} \int_{0}^{2 \pi} f_{r} r d r d \theta
$$

and

$$
A_{D L}=\frac{1}{S} \int_{0}^{R_{c}} \int_{0}^{2 \pi} r^{1-\eta} d r d \theta
$$

where $S$ is the surface of the cell. We moreover introduce the parameter Considering $n^{M S}=\rho_{M S} \pi R_{c}^{2}$ mobiles in the cell, the expression (4.1) can thus be written:

$$
P^{D L}=\operatorname{Pr}\left(n^{M S}>n_{D L}^{t h}\right)
$$

where:

$$
n_{D L}^{\text {th }}=\frac{1-\varphi}{\beta\left(\alpha+F_{D L}\right)}
$$

and as long as long as the Noise is very low, i. e.:

$$
\frac{A_{D L} \text { Noise }}{P_{\max }\left(\alpha+F_{D L}\right)}<<n_{D L}^{\text {th }}
$$

The downlink interference factor analytical expression (3.1) takes into account the network's size. We denote $c_{1}=\frac{2 R_{c}}{R}=\frac{2 R_{c}}{\left(2 N_{R}+1\right) R_{c}}=\frac{2}{2 N_{R}+1}$. This parameter represents the relative dimensions of the network compared to the distance between two localisations of BS. $N_{R}$ represents the number of rings of cells 
around the studied one. Considering $\rho_{B S}=\frac{1}{\pi R_{c}^{2}}$, we obtain, from (4.4a) and (3.1):

$$
F_{D L}=\frac{-2^{\eta+4}}{(2+\eta)} \int_{0}^{1} x^{1-\eta}\left[\left(1-\frac{x}{2}\right)^{\eta+2}-c_{1}^{-2-\eta}\left(1-c_{1} \frac{x}{2}\right)^{\eta+2}\right] \cdot d x
$$

and we notice that $F_{D L}$ is positive due to $\eta<-2$. The expression of $F_{D L}$ does not explicitly depend on the size of a cell, but only on its relative dimension to the network's one characterized by the parameter $c_{l}$. As we can observe (Table 1) the average interference factors are limited; they tend to an asymptotic value, when the network's dimension increases. For high size networks, i.e. $N_{R} \rightarrow \infty, F_{D L}$ does no more depend on the network's size: $F_{D L}=\frac{-4}{(2+\eta)} \int_{0}^{1} x^{1-\eta}(2-x)^{\eta+2} \cdot d x$. As a consequence, for a homogeneous network the number of mobiles per cell does not depend on the size of the cell. It partially depends on the environment characterized by the pathloss factor $\eta$, the SIR target, the orthogonal factor $\alpha$ and the power ratio dedicated to the common channels $\varphi$.

\subsection{Macro diversity impact}

We introduce the downlink average interference factor for the macrodiversity zone:

$$
F_{M D}=\frac{1}{S} \int_{R_{t h}}^{R_{c} 2 \pi} \int_{0}^{2 \pi}\left(\frac{1-\sigma}{1+\sigma}\right) f_{r} r d r . d \theta
$$

We moreover introduce the parameters:

$$
\Gamma_{M D}=\frac{1}{S} \int_{R_{\mu h}}^{R_{2} 2 \pi} \int_{0}^{2}\left(\frac{1-\sigma}{1+\sigma}\right) \alpha r d r \cdot d \theta
$$

and

$$
A_{M D}=\frac{1}{S} \int_{R_{t h}}^{R_{c} 2 \pi} \int_{0}^{2 \pi} r^{1-\eta} d r d \theta
$$

We can write (4.1) as:

$$
P^{D L}=\operatorname{Pr}\left(n^{M S}>n^{t h}\right)
$$

where:

$$
n^{\text {th }}=\frac{1-\varphi}{\beta\left(\alpha+F_{D L}+F_{M D}+\Gamma_{M D}\right)}
$$


and as long as long as the Noise is very low, i.e.:

$$
\frac{\left(A_{D L}+A_{M D}\right) \text { Noise }}{P_{\max }\left(\alpha+F_{D L}+F_{M D}+\Gamma_{M D}\right)}<<n^{\text {th }}
$$

The macrodiversity consequence is to decrease the term $n^{\text {th }}$ which represents the limit capacity of the cell.

\section{Macrodiversity generalization}

For the downlink, we express that the useful power received by a mobile belonging to the base station $b$ comes from $N_{M D}$ base stations of the network. Considering that any mobile is in macrodiversity with all the base stations of the network, $N_{M D}=N_{B S}$, the expression (2.1) is thus rewritten as:

$$
\frac{P_{i b} g_{i b}+\sum_{l \neq b}^{N_{B S}} P_{i l} g_{i l}}{\alpha\left(P_{b}-P_{i b}\right) g_{i b}+\sum_{l \neq b}^{N_{B S}}\left(P_{l}-P_{i l}\right) g_{i l}+N_{t h}} \geq \gamma
$$

Where $P_{i l}$ is the useful transmitting power coming from the base station 1 towards the mobile $i$ belonging to the base station $b$, and $g_{i l}$ is the path loss between the base station $l$ and the mobile $i, P_{l}$ is the total transmitting power of the base station $l$

$$
P_{i b} g_{i b}+\sum_{l \neq b}^{N_{B S}} P_{i l} g_{i l}=\gamma\left(\alpha\left(P_{b}-P_{i b}\right) g_{i b}+\sum_{l \neq b}^{N_{B S}}\left(P_{l}-P_{i l}\right) g_{i l}+N_{t h}\right)
$$

Denoting:

$$
\mu=\frac{1+\gamma}{1+\alpha \gamma}
$$

(we drop the indexes $i$ and $D L$ ), we can write:

$$
P_{i b}=\beta\left(\alpha P_{b}+f_{i} P_{b}+N_{t h} / g_{i b}\right)-\mu \frac{1}{g_{i b}} \sum_{l \neq b}^{N_{B S}} P_{i l} g_{i l}
$$

In our analysis, each base station of the network contributes to the useful power received by any mobile belonging to any base station. Due to the fact that the base stations transmitting powers are limited, and that the mobiles number in the network is great, it is reasonable to consider a limitation of the available transmitting powers $P_{i l}$ dedicated to the macrodiversity. Moreover, considering the 
great distances between the other base stations of the network and the mobile $i$ belonging to the base station $b$, we can assume that the base stations use the maximum power (denoted $P$ ) available for the transmitting power $P_{i l}$ We notice an analogy with the expression (2.2): we can write, when all the base stations transmitting powers are identical: $P_{l}=P_{b}$ for $l=1 \ldots \mathrm{N}_{\mathrm{BS}}$, and when all the transmitting powers $P_{i l}$ equal $P$ :

$$
\frac{1}{g_{i b}} \sum_{l \neq b}^{N_{B S}} P_{i l} g_{i l}=P \frac{1}{g_{i b}} \sum_{l \neq b}^{N_{B S}} g_{i l}=P f_{i}
$$

These last assumptions can be verified if the network is homogeneous, or when base stations manage a maximum number of mobiles. The total transmitting power of $\mathrm{BS}$ b can thus be written as:

$$
P_{b}=\sum_{i=1}^{N} P_{i b}+\sum_{j \neq b}^{N_{B S}} \sum_{i=1}^{N} P+P_{C C H}
$$

or

$$
P_{b}=\sum_{i=1}^{N} P_{i b}+N\left(N_{B S}-1\right) P+P_{C C H}
$$

and finally:

$$
\sum_{i=1}^{N} P_{i b}=\sum_{i=1}^{N} \beta\left(\alpha P_{b}+f_{i} P_{b}+N_{t h} / g_{i b}\right)-\mu \sum_{i=1}^{N} P f_{i}
$$

Considering that the power $P$ is a fraction of the total power $P_{b}$, we can write, $P=\varepsilon$ $P_{b}$, and when:

$$
P_{c c h}=\varphi P_{b} \sum_{i=1}^{N} P_{i b}=N \beta \alpha P_{b}+(\beta-\mu \varepsilon) P_{b} \sum_{i=1}^{N} f_{i}+\sum_{i=1}^{N} \beta N_{t h} / g_{i b}
$$

and

$$
P_{b}=N \beta \alpha P_{b}+(\beta-\mu \varepsilon) P_{b} \sum_{i=1}^{N} f_{i}+\sum_{i=1}^{N} \beta N_{t h} / g_{i b}+N\left(N_{B S}-1\right) \varepsilon P_{b}+\varphi P_{b}
$$

So we have:

$$
P_{b}\left(1-\varphi-N \beta \alpha-(\beta-\mu \varepsilon) \sum_{i=1}^{N} f_{i}-N\left(N_{B S}-1\right) \varepsilon\right)=\sum_{i=1}^{N} \beta N_{t h} / g_{i b}
$$

Using our analytical model we can write: $\sum_{i=1}^{N} f_{i}$ as $\int_{0}^{R_{c}} \int_{0}^{2 \pi} \rho_{M S} f_{r} r d r d \theta$

Denoting: 


$$
F=\frac{1}{S_{\text {cell }} S_{\text {Network }}} \int_{0}^{R_{c} 2 \pi} \int_{0}^{2 \pi} \frac{2 \pi}{(\eta+2) r^{\eta}} \cdot\left[(R-r)^{\eta+2}-\left(2 R_{c}-r\right)^{\eta+2}\right] r d r d \theta
$$

we can write $P_{b}\left(1-\varphi-N_{M S} \beta \alpha-(\beta-\mu \varepsilon) N_{M S} N_{B S} F-N_{M S}\left(N_{B S}-1\right) \varepsilon\right)=\sum_{i=1}^{N} \beta N_{t h} / g_{i b}$ and express $P_{b}$ as:

$$
P_{b}=\frac{\sum_{i=1}^{N} \beta N_{t h} / g_{i b}}{1-\varphi-N_{M S}\left(\beta \alpha-(\beta-\mu \varepsilon) N_{B S} F-\left(N_{B S}-1\right) \varepsilon\right)}
$$

The denominator has to be positive:

$1-\varphi-N_{M S}\left(\beta \alpha-(\beta-\mu \varepsilon) N_{B S} F-\left(N_{B S}-1\right) \varepsilon\right)>0$

So the cell capacity is given by:

$$
N_{M S}=\frac{1-\varphi}{\beta \alpha+(\beta-\mu \varepsilon) N_{B S} F+\left(N_{B S}-1\right) \varepsilon}
$$

We denote $N_{l, M S}$ the cell capacity without macrodiversity, i.e. $\varepsilon=0$ :

$$
N_{1, M S}=\frac{1-\varphi}{\beta \alpha+\beta N_{B S} F}
$$

Does the macrodiversity increase the capacity of a cell? To answer that question we compare (4.9) and (4.10):

$$
\frac{1-\varphi}{\beta \alpha+(\beta-\mu \varepsilon) N_{B S} F+\left(N_{B S}-1\right) \varepsilon}>\frac{1-\varphi}{\beta \alpha+\beta N_{B S} F} \text { which can be written as }
$$
$\beta \alpha+(\beta-\mu \varepsilon) N_{B S} F+\left(N_{B S}-1\right) \varepsilon<\beta \alpha+\beta N_{B S} F$ and finally, denoting

$$
N_{B S, t h}=\frac{1}{1-\mu F}
$$

we conclude that macrodiversity increases the capacity only if we have:

$$
N_{B S}<N_{B S, t h}
$$

For $\eta=-3, R_{c}=1, R=10$, we obtain $\mathrm{F}=0.72 \%$.

For $\alpha=0.7$ and $\gamma=-16 \mathrm{dBm}$ (voice service), we have $N_{B S, t h}=1.007$. The downlink macrodiversity decreases the capacity of a cell. We can observe that result whatever the values of $\mathrm{F}$. The loss of capacity due to macrodiversity is Loss = $\mathrm{N}_{1 \mathrm{MS}}-\mathrm{N}_{\mathrm{MS}}$ 


$$
\begin{aligned}
& \text { Loss }=\frac{1-\varphi}{\beta \alpha+\beta N_{B S} F}-\frac{1-\varphi}{\beta \alpha+(\beta-\mu \varepsilon) N_{B S} F+\left(N_{B S}-1\right) \varepsilon} \\
& =(1-\varphi) \frac{(-\mu \varepsilon) N_{B S} F+\left(N_{B S}-1\right) \varepsilon}{\left(\beta \alpha+\beta N_{B S} F\right)\left(\beta \alpha+(\beta-\mu \varepsilon) N_{B S} F+\left(N_{B S}-1\right) \varepsilon\right)}
\end{aligned}
$$

Figures 2 and 3 show the loss of capacity in term of mobile number (figure 2: orange curve) and loss percentage of cell capacity (figure3: violet curve) as a function of the percentage of power $\varepsilon$ dedicated to each link for the macrodiversity. The red curve shows the total transmitting power of a base station, dedicated to mobiles in macrodiversity.

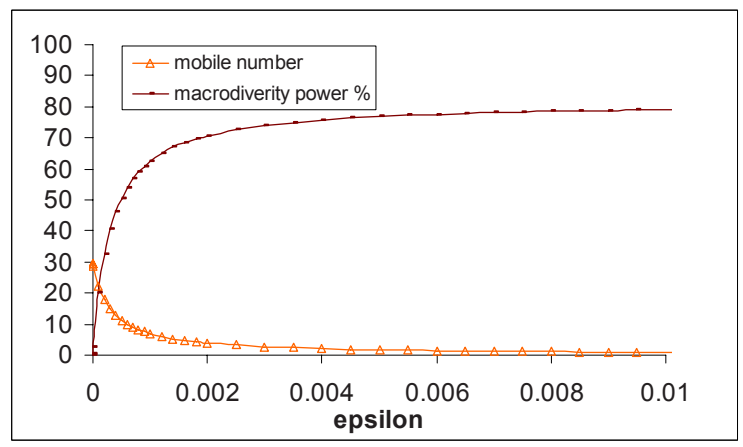

Fig. 2. Macrodiversity impact on the cell capacity vs percentage of transmitting power

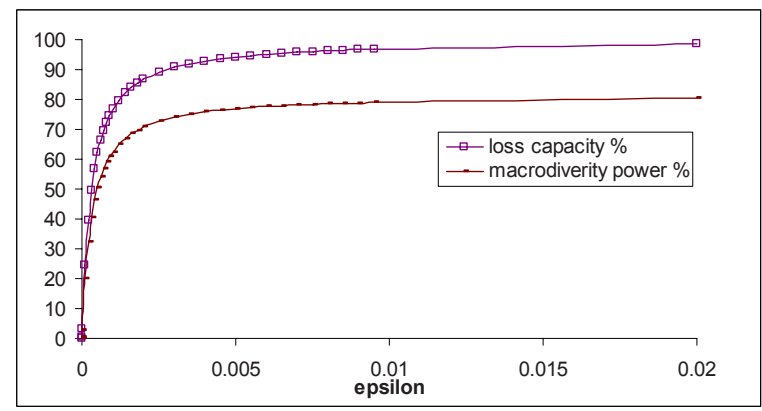

Fig. 3. Macrodiversity impact on the loss of capacity vs percentage of transmitting power

\section{Cancellation of the other cell interferences}

We notice from (4.6b) that the term $f_{i}$ vanishes for $\beta=\mu \varepsilon$, as if the interferences felt by a mobile, due to the other base stations of the network, were balanced by the fraction of their transmitting powers dedicated to that mobile. Since 
$\beta=\frac{\gamma}{1+\alpha \gamma}$ and $\mu=\frac{1+\gamma}{1+\alpha \gamma}$, we have $\varepsilon=\frac{\gamma}{1+\gamma}$ The cell capacity $\mathrm{N}_{1, \mathrm{MS}}$ can be written:

$$
N_{1, M S}=\frac{1-\varphi}{\beta \alpha}
$$

\section{Conclusion}

In this paper, we established an explicit expression of the load $L_{M D}$ due to mobiles in macrodiversity, and showed that one increases the cell's load. We identified the traffic and network's parameters which play a key role in the cell's load. We finally generalized the approach, considering a macrodiversity with a great number of base stations. Though mainly focused on CDMA networks, our analysis can be applied to any frequency reuse 1 network based on other technologies, such as OFDMA.

\section{References}

1. A. J. Viterbi, CDMA Principles of Spread Spectrum Communication, Wesley, 1995.

2. T. Bonald and A. Proutiere, Wireless Downlink Data Channels: User Performance and Cell Dimensioning, ACM Mobicom 2003

3. Jaana Laiho, Achim Wacker Tomas Novosad, "Radio network planning and optimisation for UMTS"

4. Hiltunen, K., De Bernardi, R. WCDMA Downlink capacity estimation, VTC 2000, p. 992996

5. F. Baccelli, B. Błaszczyszyn, and F. Tournois (2003) Downlink admission/congestion control and maximal load in CDMA networks, in Proc. of IEEE INFOCOM'03

6. H. Holma A. Toskala, WCDMA for UMTS, Radio Access for Third Generation Mobile Communications. John Wiley \& Sons, Ltd.,2001.

7. S.V Hanly,., "Capacity and Power Control in Spread Spectrum Macrodiversity Radio Networks”, IEEE Trans. on Comm., vol. 44, NO. 2, pp.247-256, Feb. 1996

8. D. Aktas, M. N. Bacha, J. S. Evans, and S. V. Hanly, Scaling Results on the Sum Capacity of Cellular Networks With MIMO Links, IEEE transactions on information theory, vol 52, $\mathrm{n}^{\circ} 7$, July 2006

9. J-Marc Kelif and E. Altman, Downlink fluid model for CDMA Networks, VTC 2005 Stockholm

10. P. Jacquet, "Geometry of information propagation in massively dense adhoc networks," in Proc. ACM MobiHOC, Roppongi Hills, Japan, May 2004, pp. 157-162.

11. J-Marc Kelif, M. Coupechoux, and P. Godlewski, Spatial Outage Probability for Cellular Networks, Globecom 2007, Washington 